\title{
Transcatheter tricuspid valve repair: Bringing the forgotten valve into the spotlight
}

Marissa Donatelle, MD, and Gorav Ailawadi, MD, MBA

Feature Editor's Note-Ailawadi and colleagues have provided an excellent summary of the spectrum of transcatheter devices for the treatment of tricuspid regurgitation (TR). Functional TR is the most common form of TR, and it remains largely asymptomatic until severe enough to cause right heart failure or congestive symptoms. Severe symptomatic TR will often not be treated surgically because of the associated high morbidity, in-hospital mortality, and poor long-term survival associated with open repair or replacement. Transcatheter approaches for the treatment of severe TR are ideal for those patients with severe, symptomatic TR, a high surgical risk, severe right ventricular dysfunction, or severe pulmonary hypertension. With the development of these new approaches, patients with multivalve disease can now be approached in a manner more similar to that for surgery, without leaving the pathology untreated. Although transcatheter solutions in the aortic and mitral realm have shown success, several factors have led to difficulties with transcatheter approaches for tricuspid valve disease. The complexity of the tricuspid valve anatomy such as its thinner leaflets, its elliptical annular shape, and, rarely, the presence of calcified structures has made the technology used for the aortic valve and mitral valve less reproducible for use with the tricuspid valve. Another important factor has been the difficulty with intraprocedural imaging guidance-the leaflets can be more difficult to see, grasping them can be difficult to accurately image, and shadowing from any mitral or aortic prostheses can significantly impair tricuspid valve visualization. Despite these limitations, several tricuspid valve-specific transcatheter solutions have been developed. As surgeons who have experience with tricuspid surgery, it is important that we remain engaged and lead in this area. The present summary details the variety of devices on the market used to treat TR percutaneously; they fall into 2 broad

\footnotetext{
From the Department of Surgery, University of Virginia School of Medicine, Charlottesville, Va.

Received for publication Sept 23, 2019; revisions received March 8, 2020; accepted for publication April 2, 2020; available ahead of print June 26, 2020.

Address for reprints: Gorav Ailawadi, MD, MBA, Department of Surgery, University of Virginia School of Medicine, 1215 Lee St, Charlottesville, VA 22908 (E-mail: gorav@virginia.edu).

J Thorac Cardiovasc Surg 2020;160:1467-73

$0022-5223 / \$ 36.00$

Copyright $(2020$ by The American Association for Thoracic Surgery

https://doi.org/10.1016/j.jtcvs.2020.04.184
}

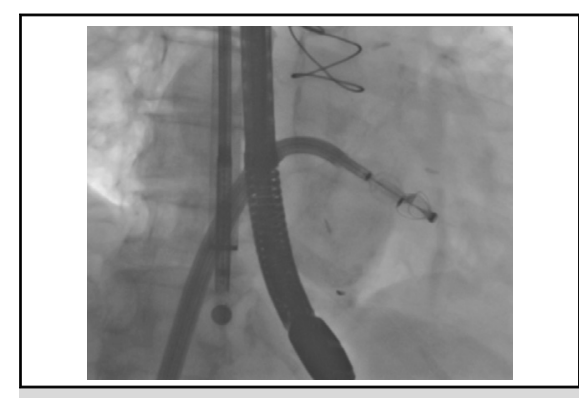

Fluoroscopic picture of 1 Pascal (Edwards LifeSciences, Irvine, Calif) implanted in the tricuspid position, Pascal Delivery system, intrajugular pediatric echo, and Transesophageal echo.

\section{CENTRAL MESSAGE \\ The field of transcatheter \\ tricuspid valve repair is rapidly \\ growing. We examined the cur- \\ rent state of transcatheter \\ tricuspid repair as an isolated \\ intervention, as well as in \\ combination.}

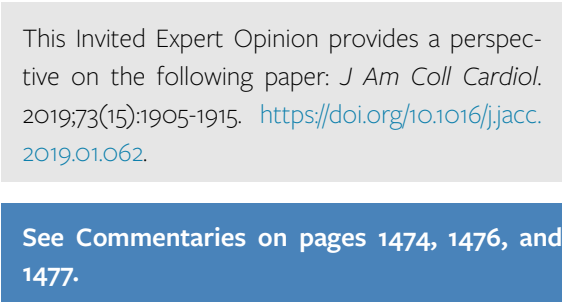

categories-those that aid in coaptation and those that replicate surgical annuloplasty.

\section{Alison F. Ward, MD}

\section{Richard Lee, MD, JD MBA}

Once nicknamed the "forgotten valve," the tricuspid valve (TV) has finally garnered the attention it is due. At least 1.6 million Americans are affected by severe tricuspid regurgitation (TR), and this number is likely underrepresented. ${ }^{1}$ Currently, the prevalence of TR is as high as $5.6 \%$ in men $>70$ years old. ${ }^{2,3}$ Furthermore, with the aging population, the burden of TR can be 
expected to increase during the next decade. Although $>80 \%$ of TR arises from a functional mechanism (FTR), TR can also arise from organic/primary valve disease, commonly from pacer leads affecting leaflet motion. FTR can result from advanced left side disease, pulmonary hypertension, right ventricular dilation, or right atrial dilation, all of which can lead to tricuspid annular dilation. ${ }^{4}$ Compared with the left side of the heart, the right side has unique challenges. Because of its lower profile musculature in the right ventricle (RV), little room is available to resist and remodel with the increase in fluid and stretch that accompanies pulmonary hypertension. Early right-sided heart failure can quickly progress to massive annular dilation. When left untreated, TR will result in worsening right-sided heart failure, resulting in ascites, peripheral edema, and fatigue, with patients requiring frequent hospitalizations and experiencing a poor quality of life.

The current guidelines from the American Heart Association/American College of Cardiology have stated that surgical repair of severe, symptomatic TR should be the gold standard. However, because these patients will often be referred late, they will commonly present with advanced RV dysfunction, rendering them highrisk candidates for surgery. ${ }^{5}$ The challenge lies in that TR is clinically silent during the early and middle stages of the disease and that once TR is severe enough to cause right heart failure symptoms, intervention comes with greater risk in the setting of advanced RV dysfunction. $^{6}$ As such, patients with TR must be identified earlier and referred to reference centers specializing in TV surgical as well as transcatheter approaches for assessed by a multidisciplinary heart team before extreme annular dilation and right-sided heart failure symptoms have developed.

Medical therapy is a critical component in the care of patients with TR. Similar to the mitral valve, FTR has been shown to be positively affected by heart failure medications. However, patients will often remain symptomatic despite optimal medical therapy (OMT), leaving nonsurgical patients with few options until recently. Although in the past few years, a surge has occurred in the development of transcatheter tricuspid repair devices, which have shown promise as options for these previously untreated patients, challenges remain with imaging the TV. Once imaging of the TV has improved, so will the technologies and outcomes. As of January 2020, all these devices are under clinical trial in the United States, and most have excluded the subpopulation of patients with pacemaker lead-induced TR. Although the goals are to increase leaflet coaptation, these devices, in general, can be placed into 2 categories according to their mechanism of action: (1) direct leaflet apposition, or (2) annular reduction.

\section{COAPTATION ASSISTANCE DEVICES MitraClip System}

The MitraClip system (Abbott Laboratories, Santa Clara, Calif) was first developed to simulate an edge-to-edge Alfieri-stitch technique in the setting of mitral regurgitation (MR). ${ }^{7}$ Using a $24 \mathrm{~F}$ delivery system, the MitraClip is introduced via transfemoral venous access under transesophageal echocardiography (TEE) and fluoroscopic guidance. The ACCESS-EU (ACCESS-Europe: a two-phase observational study of the MitraClip System in Europe), EVEREST (endovascular valve edge-to-edge repair study), and COAPT (cardiovascular outcomes assessment of the MitraClip percutaneous therapy for heart failure patients with functional MR) trials were fundamental to the approval of the MitraClip in both Europe (2008) and the United States (degenerative MR in 2013 and functional MR in 2019) for the treatment of MR. ${ }^{8-11}$

In 2016, Hammerstingl and colleagues ${ }^{12}$ described the successful use of the MitraClip system in the tricuspid position using transjugular access. Soon thereafter, a transition occurred to the use of a transfemoral approach, allowing for easier device steering. Following the first account of the MitraClip in the tricuspid position, Nickenig and colleagues ${ }^{13}$ reported the largest series to date, with 64 cases from 10 hospitals throughout the world in 2017. They had great site-reported success, with a reduction in TR in $94 \%$ of the cases and improvement in New York Heart Association (NYHA) function class and 6-minute walk test distance. The in-hospital mortality rate for these patients was $5 \%$, primarily because these patients had been extremely sick with advanced heart failure before intervention. ${ }^{13}$

Recently, Abbott Laboratories began an investigation of a modified MitraClip device designed specifically for the TV in a clinical trial (Figure 1). This system has altered steering knobs from the commercially available device and also includes the slightly larger MitraClip XTR to accommodate the larger distance between leaflets often seen in TR. The TRILUMINATE (trial to evaluate treatment with Abbott transcatheter clip repair system in patients with moderate or greater tricuspid regurgitation) was an early feasibility study (EFS) international single-arm feasibility trial that demonstrated $\geq 1$ grade of reduction in TR in $86 \%$ of patients at 1 month, with an all-cause mortality of $5 \%$ at 6 months. ${ }^{14}$ The multicenter TRILUMINATE pivotal trial has just begun enrollment, which will randomize 700 patients at 66 sites to the tricuspid MitraClip plus OMT versus OMT alone. ${ }^{15}$ 


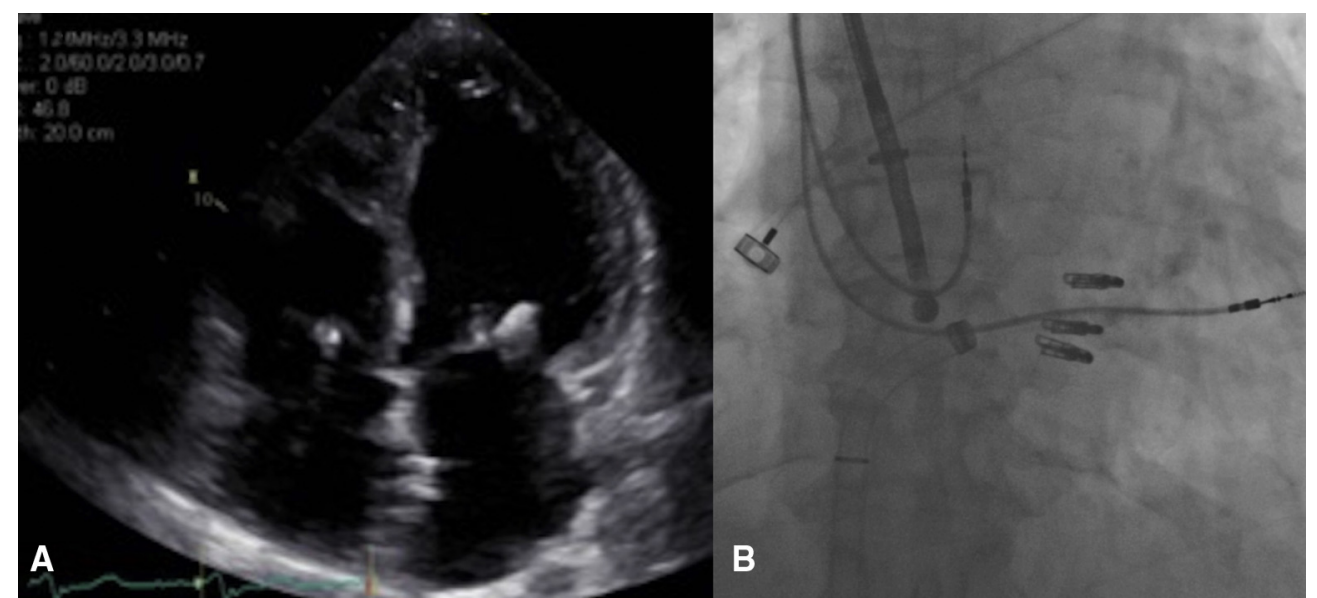

FIGURE 1. A, Transthoracic echocardiography of a patient with previous MitraClip in the mitral position following off-label implantation of 2 MitraClips (Abbott, Santa Clara, Calif) in the tricuspid position. B, Fluoroscopic image of 3 implanted MitraClips in the tricuspid position.

\section{PASCAL Device}

The PASCAL (paddle, spacer, clasps, Alfieri stitch; Edwards Lifesciences, Irvine, Calif) device was first implanted in December 2016 in the mitral position. Similar to other direct repair systems, it is delivered via a transfemoral approach. What made the PASCAL device distinct from the original MitraClip device is that it has 2 independently clasping arms that are slightly larger, with a spacer in the middle (Figure 2). ${ }^{16}$ The spacer is placed between 2 valve leaflets, catching the leaflets independently and clasping them together. First-in-human and feasibility trials for PASCAL to treat MR were completed in Europe with good results. In the first reported results from the compassionate use of this device in the mitral position, $96 \%$ of the 22 patients treated had $\leq 2+$ MR. ${ }^{17}$ In the recently reported results from the first-in-human experience in the tricuspid position, all 28 patients treated were considered to have a high surgical risk, and $92 \%$ had FTR. Procedural success occurred in $86 \%$ of the patients with no intraprocedural complications. At the 30-day follow-up, $88 \%$ of the patients had had a NYHA class of I or II and $85 \%$ of the patients had had a TR grade of $\leq 2+.{ }^{18}$ Given these results,
PASCAL is currently available under clinical trial for TR in the early feasibility CLASP TR trial (Edwards CLASP TR EFS) with enrollment soon to begin in a randomized pivotal CLASP TR trial comparing OMT to PASCAL plus OMT. ${ }^{19}$ A few potential advantages of PASCAL for the treatment of TR include the larger implant size, spacer, and long paddles, allowing for easier positioning. Although a few cases have been performed, no data have been reported to date on the use of PASCAL in the tricuspid position.

\section{Forma Device}

The Forma (Edwards Lifesciences) is another leaflet apposition device that had shown initial promise. This device differs from other devices in that it is a foam-filled spacer that is positioned at the level of the TV and fixed on a rail that is secured to the apex of the RV by a nitinol anchor (Figure 3). ${ }^{20}$ The spacer allows for an additional surface area by which the tricuspid leaflet can coapt against. The device is implanted through the left subclavian vein, across the right atrium and TV, and anchored to the right ventricular septum, using TEE and fluoroscopic guidance.

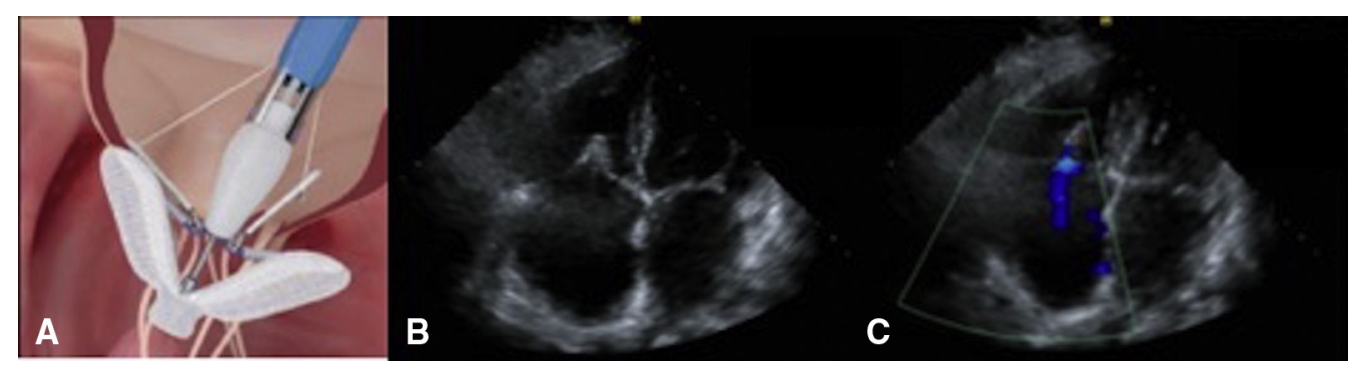

FIGURE 2. A, Artist rendering of PASCAL (Edwards LifeSciences, Irvine, CA, USA) device with one of two grippers deployed. ${ }^{15}$ B and C, Transthoracic images of a patient following PASCAL deployment. 


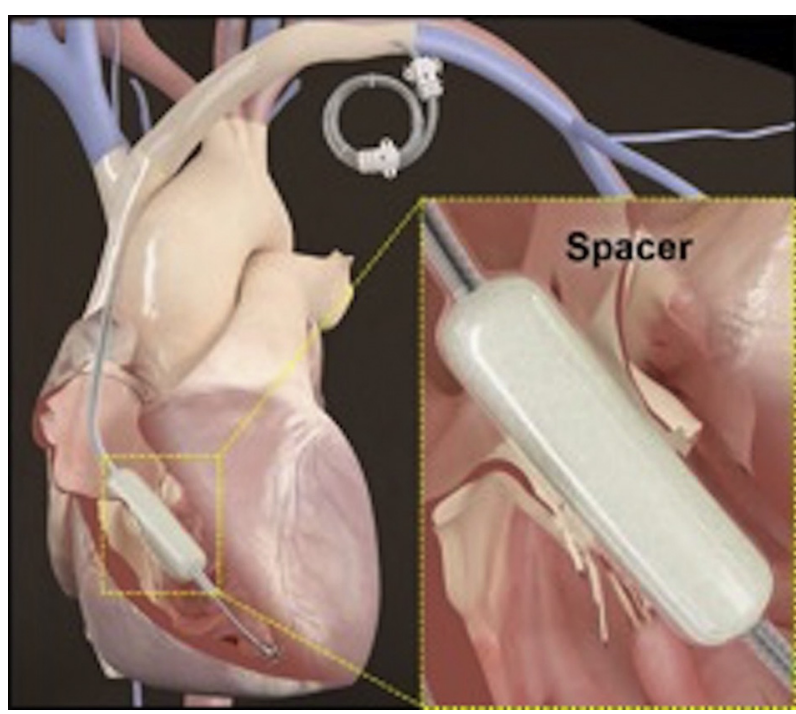

FIGURE 3. Artist rendering of the FORMA Repair System (Edwards Lifesciences, Irvine, Calif) anchored in the right ventricular apex and positioned at the annulus of the tricuspid valve. ${ }^{18}$

In 2015, Campelo-Parada and colleagues ${ }^{21}$ reported the first-in-human study of 7 patients. Procedural success was seen in $85 \%$ of patients. At 1 year of follow-up, promising quality of life results were found, with sustained improvement in the 6-minute walk test distance, NYHA functional class, and quality of life survey results in all patients with successful implantation. ${ }^{21}$ In 2018, Perlman and Dvir $^{20}$ reported preliminary early feasibility results for 29 patients from 5 sites. At baseline, $86 \%$ of the patients had had NYHA functional class of $\geq$ III, which had improved greatly to only $28 \%$ at 30 days after implantation. ${ }^{20}$ Despite these initial promising results, Edwards Lifesciences has pulled the device from trials because of issues with anchoring in the RV and movement of the device that varied with patient positioning.

\section{ANNULOPLASTY DEVICES}

\section{Cardioband Direct Annuloplasty}

The Cardioband device (Edwards Lifesciences) was developed to mimic surgical tricuspid repair by implanting a C-shaped polyester sleeve anchored along the tricuspid annulus (Figure 4). ${ }^{22}$ Extensive preoperative planning, including implant size selection, is performed using computed tomography and TEE. The delivery system uses a $25 \mathrm{~F}$ sheath in the femoral vein. Between 12 and 17 adjustable and retrievable steel anchors are used to secure the Cardioband in the tricuspid annulus under fluoroscopic and TEE guidance. Selective right coronary angiography has been frequently performed to ensure avoidance of coronary injury with the anchors, which was documented in roughly $10 \%$ of early cases. On release of the final anchor, an adjustment tool is used to gradually cinch the Cardioband and tricuspid annulus in a stepwise fashion. ${ }^{23}$

Similar to other tricuspid repair devices, Cardioband also found success first in the mitral position and received CE Mark approval. The results from the EFS trial for function MR reported in 2015 showed MR $\leq 2+$ in $90 \%$ of patients at 1 year and improvement in the quality of life metrics. ${ }^{24}$

Not long after the first-in-human implantation in the tricuspid position had occurred in 2017 , numerous compassionate use implantations had been performed, with enrollment of patients into the EFS (TRI-REPAIR [tricuspid regurgitation repair with Cardioband transcatheter system]) trial for tricuspid regurgitation in the United States starting in $2018 .{ }^{25,26}$ No results from the study have been reported to date. However, in the CE Mark study of the same name, TRI-REPAIR, the 6-month follow-up results have been

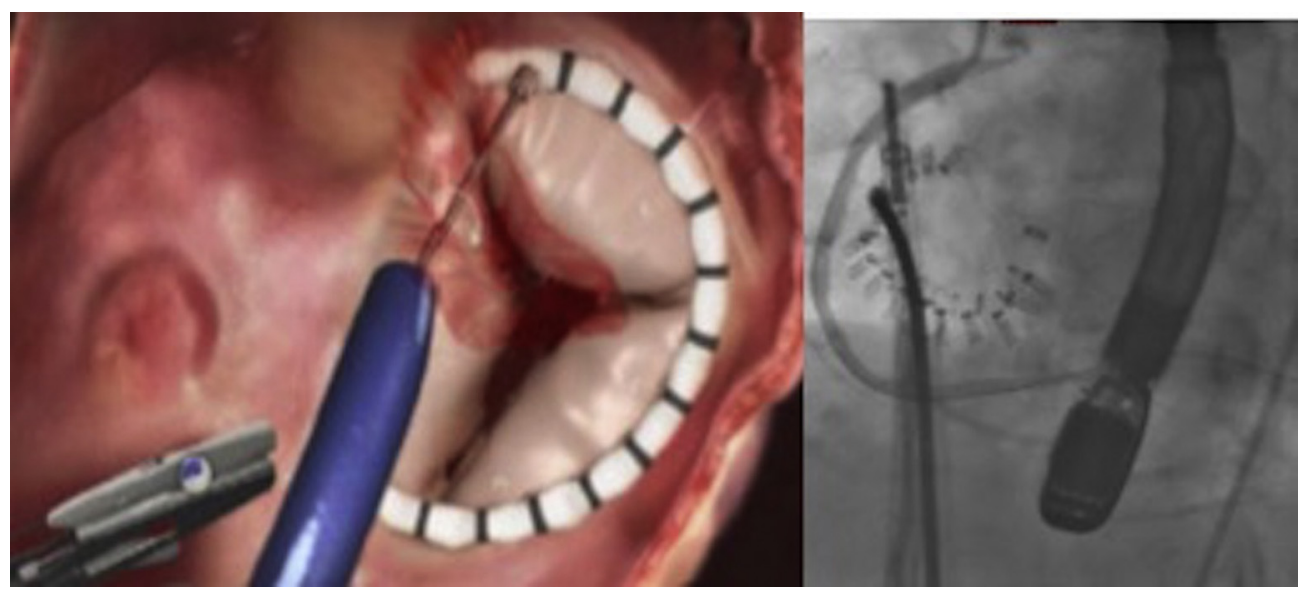

FIGURE 4. Artist rendering of Cardioband (Edwards Lifesciences, Irvine, Calif) adjustment after implantation on tricuspid annulus $(l e f t) .{ }^{20}$ Fluoroscopic image of a patient at The University of Virginia post cinch in the setting of Cardioband TR (right). 


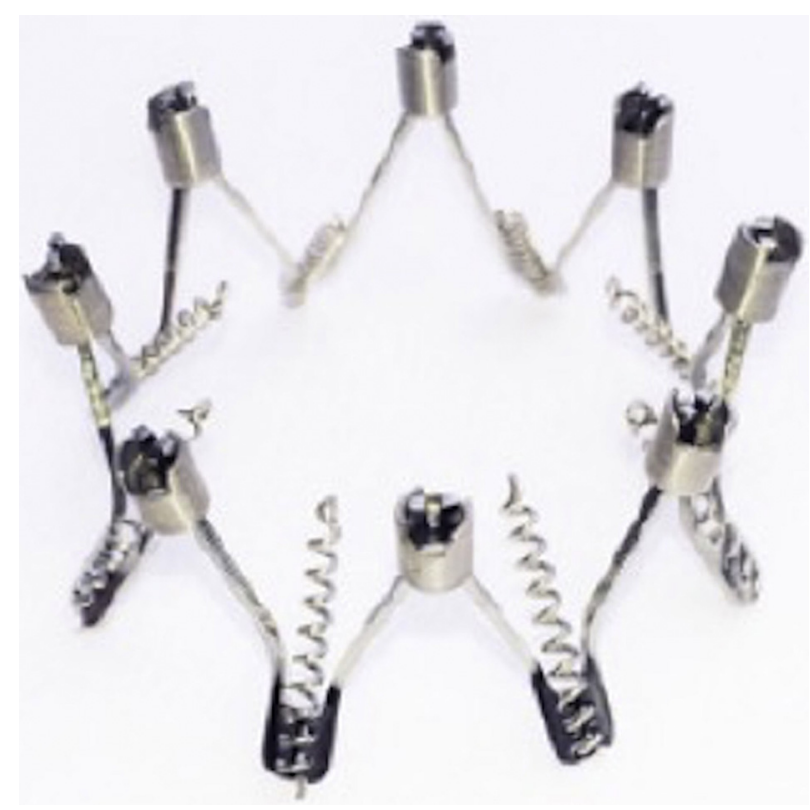

FIGURE 5. Photograph of Millipede (Boston Scientific, Marlborough, Mass) direct annuloplasty ring. ${ }^{25}$

promising. At 6 months, a statistically significant, albeit modest, $9 \%$ annular diameter reduction from 42 to $38 \mathrm{~mm}$ was found $(P<.01)$. More importantly, favorable quality of life improvement was seen, with $>80 \%$ of patients reporting a NYHA functional status of $\leq$ II. However, of the 30 patients enrolled, 3 had died at 6 months, 1 of which was related to the Cardioband device. In the latter, the death was believed to be the result of a right coronary artery branch occlusion that had occurred intraprocedurally, resulting in RV decompensation. At the time of the procedure, this occlusion had not been thought to be significant and was not treated. Despite these concerns, use of the Cardioband has shown promise in the treatment of FTR but has been limited by the challenging intraprocedural imaging. . $^{2,27}$

\section{Millipede IRIS}

The Millipede IRIS (Boston Scientific, Marlborough, Mass) uses a transvenous approach to implant a collapsible nitinol zigzag-shaped semirigid circumferential annular ring (Figure 5). ${ }^{28}$ The IRIS system consists of the annular ring, 7 to 9 screw anchors, and collars. The ring is expanded, and anchors at each of the inferior zigzags are used to secure the ring in a supra-annular position. The collars, which are positioned on the superior zigzags, are moved ventricularly, cinching the ring and reducing the size of the annulus $\leq 50 \%$. The IRIS is repositionable and adjustable to allow for proper sizing and positioning. The zone of coaptation is measured

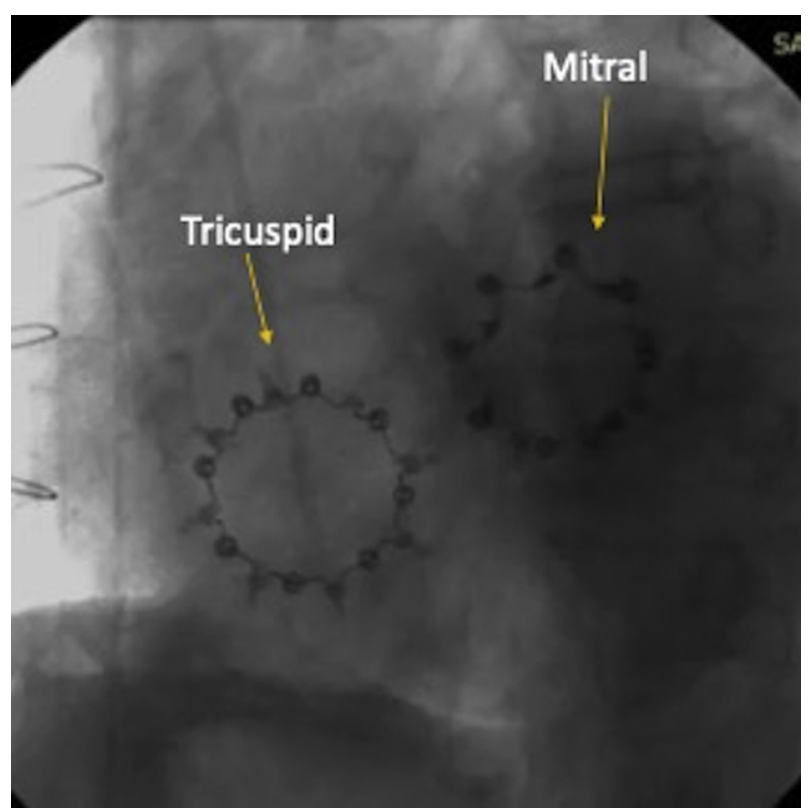

FIGURE 6. Fluoroscopic image of implanted Millipede IRIS (Boston Scientific, Marlborough, Mass) in both the mitral and tricuspid position. ${ }^{27}$

using intracardiac echosonography, which is mounted on the delivery system, during the cinching. In 2015, the first-in-human procedure was performed via an open surgical approach for use in the mitral position, followed by a successful transcatheter approach in April 2017. To the best of our knowledge, $\geq 32$ Millipede implantations have been performed to date in the mitral position. ${ }^{29}$ In 2016, Rogers ${ }^{30,31}$ presented promising results from a small cohort of patients who had received the Millipede device in the tricuspid position (Figure 6). These cases had included combined mitral and tricuspid implantation, using the same implant. In the tricuspid position, only 7 of the 9 anchors were implanted to avoid atrioventricular node injury. At baseline, all the patients had severe TR and NYHA functional class IV. The results from this small cohort were encouraging, with 2 patients maintaining no or mild TR at 3 years. No pacemakers have been required. An EFS (annular reshaping of the mitral valve for patients with mitral regurgitation using the Millipede IRIS system) for the mitral position, but not excluding concurrent tricuspid implantation, began enrolling in 2017 , with no results reported to date. ${ }^{32}$

\section{Summary}

The world of transcatheter tricuspid repair devices has rapidly advanced in the past few years and is expected to exponentially expand with the increased investment in development of additional tricuspid-specific devices. The implantation of a transcatheter direct annuloplasty 


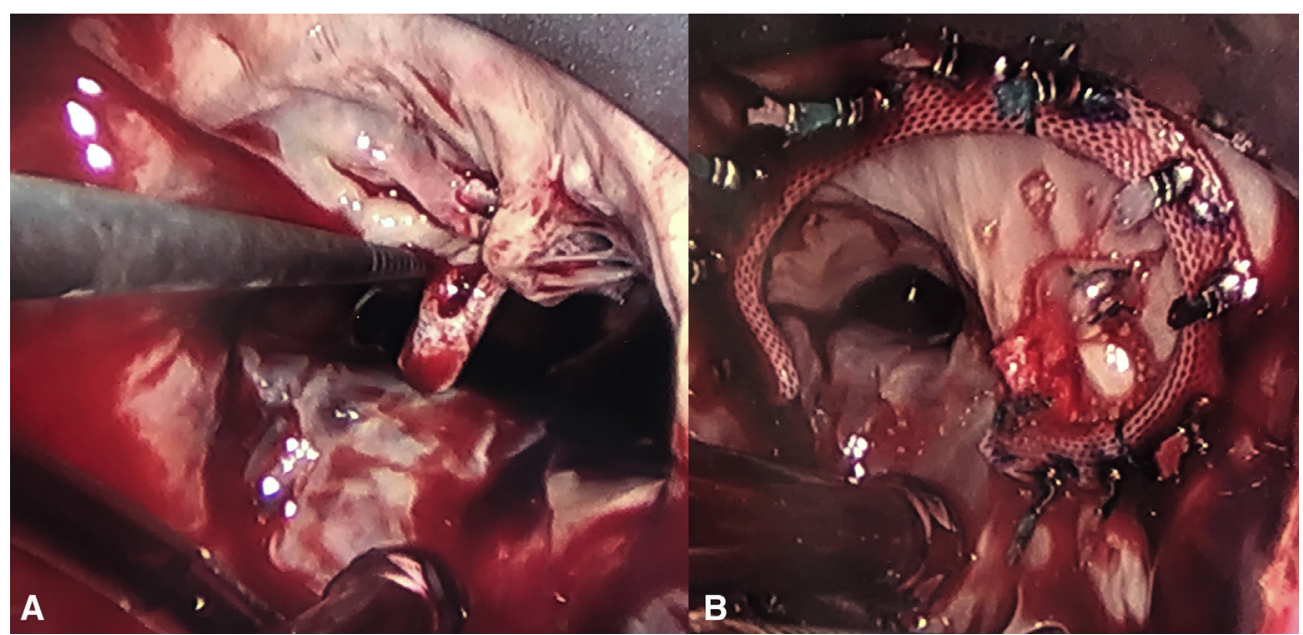

FIGURE 7. Intraoperative image of removal of failed MitraClip in the tricuspid position (A) and surgical repair with a ring (B).

has opened the door for future transcatheter valve-in-ring options should the need arise. However, the direct coaptation apposition devices do not allow for the opportunity for future transcatheter valve replacement, although they do have the option of combining with annuloplasty devices to augment the coaptation. Although not the focus of the present report, transcatheter TV replacement has been slow to develop owing to (1) the large nature of the tricuspid annulus in patients with TR, and (2) the challenges with leaflet/annular fixation. Great investment has occurred for transcatheter TV replacement, with some promising compassionate use cases with the Navia (NaviGate Cardiac Structures Inc, Lake Forest, Calif) and Evoque (Edwards Lifesciences, Irvine, Calif) valves primarily for patients in whom the described repair approaches are not feasible anatomically. ${ }^{33}$

\section{CONCLUSIONS}

The TV is no longer forgotten and has been put in the spotlight. Transcatheter technologies have augmented our interest in treating the TV both surgically and with a catheter. This new found interest in treating the TV has resulted in a better understanding of the mechanics of the valve as well as RV and has brought to light the need for growth in imaging technology. With this increasing knowledge, it is evident that patients with significant TR must be identified earlier and referred to reference centers with expertise in the medical, surgical, as well as transcatheter approaches for treating the TV specifically before the onset of torrential tricuspid regurgitation or severe right-sided heart failure symptoms. Surgeon involvement is paramount in the tricuspid heart team as it pertains to surgical risk and should be integral members of implantation teams for these novel transcatheter devices, performing complex tricuspid surgical operations or when transcatheter approaches fail (Figure 7). ${ }^{34}$ As therapies evolve, we will soon be faced with determining how to best correct TR. Whether to repair surgically or with a single or combination of transcatheter devices definitively, future investigations are required to determine how best to optimize patient selection when approaching the expanding tricuspid armamentarium.

\section{Conflict of Interest Statement}

Ailawadi reported consultancy for Abbott, Medtronic, Edwards, Gore, Admedus, and Atricure. Donatelle reported no conflicts of interest.

The Journal policy requires editors and reviewers to disclose conflicts of interest and to decline handling or reviewing manuscripts for which they may have a conflict of interest. The editors and reviewers of this article have no conflicts of interest.

\section{References}

1. Stuge O, Liddicoat J. Emerging opportunities for cardiac surgeons within structural heart disease. J Thorac Cardiovasc Surg. 2006;132:1258-61.

2. Buzzatti N, De Bonis M, Moat N. Anatomy of the tricuspid valve, pathophysiology of functional tricuspid regurgitation, and implications for percutaneous therapies. Interv Cardiol Clin. 2018;7:1-11.

3. Singh JP, Evans JC, Levy D, Larson MG, Freed LA, Fuller DL, et al. Prevalence and clinical determinants of mitral, tricuspid, and aortic regurgitation (the Framingham heart study). Am J Cardiol. 1999;83:897-902.

4. Rodés-Cabau J, Hahn RT, Latib A, Laule M, Lauten A, Maisano F, et al. Transcatheter therapies for treating tricuspid regurgitation. J Am Coll Cardiol. 2016; 67:1829-45.

5. Nishimura RA, Otto CM, Bonow RO, Carabello BA, Erwin JP III, Guyton RA, et al. 2014 AHA/ACC guideline for the management of patients with valvular heart disease. J Thorac Cardiovasc Surg. 2014;148:e1-132.

6. Van Rosendael PJ, Delgado V, Bax JJ. The tricuspid valve and the right heart: anatomical, pathological and imaging specifications. EuroIntervention. 2015; 11:W123-7. 
7. Alfieri O, Maisano F, De Bonis M, Stefano PL, Torracca L, Oppizzi M, et al. The double-orifice technique in mitral valve repair: a simple solution for complex problems. J Thorac Cardiovasc Surg. 2001;122:674-81.

8. Mendirichaga R, Singh V, Blumer V, Rivera M, Rodriguez AP, Cohen MG, et al. Transcatheter mitral valve repair with MitraClip for symptomatic functional mitral valve regurgitation. Am J Cardiol. 2017;120:708-15.

9. Stone GW, Lindenfeld J, Abraham WT, Kar S, Lim DS, Mishell JM, et al. Transcatheter mitral-valve repair in patients with heart failure. N Engl J Med. 2018; 379:2307-18

10. Glower DD, Kar S, Trento A, Lim DS, Bajwa T, Quesada R, et al. Percutaneous mitral valve repair for mitral regurgitation in high-risk patients: results of the EVEREST II study. J Am Coll Cardiol. 2014;64:172-81.

11. Maisano F, Franzen O, Baldus S, Schäfer U, Hausleiter J, Butter C, et al. Percutaneous mitral valve interventions in the real world: early and 1-year results from the ACCESS-EU, a prospective, multicenter, nonrandomized post-approval study of the MitraClip therapy in Europe. J Am Coll Cardiol. 2013;62:1052-61.

12. Hammerstingl C, Schueler R, Malasa M, Werner N, Nickenig G. Transcatheter treatment of severe tricuspid regurgitation with the MitraClip system. Eur Heart J. 2016;37:849-53.

13. Nickenig G, Kowalski M, Hausleiter J, Braun D, Schofer J, Yzeiraj E, et al. Transcatheter treatment of severe tricuspid regurgitation with the edge-to-edge MitraClip technique. Circulation. 2017; 135:1802-14.

14. Nickenig G, Weber M, Lurz P, von Bardeleben RS, Sitgers M, Sorajja P, et al. Transcatheter edge-to-edge repair for reduction of tricuspid regurgitation: 6-month outcomes of the TRILUMINATE single-arm study. Lancet. 2019;394: 2002-11.

15. Evaluation of Treatment With Abbott Transcatheter Clip Repair System in Patients With Moderate or Greater Tricuspid Regurgitation (TRILUMINATE). Available at: https://clinicaltrials.gov/ct2/show/NCT03227757. Accessed August 19, 2019.

16. Edwards PASCAL transcatheter valve repair system. Available at: https:// www.edwards.com/gb/devices/transcatheter-valve-repair/PASCAL. Accessed August 19, 2019.

17. Praz F, Spargias K, Chrissoheris M, Büllesfeld L, Nickenig G, Deuschl F, et al. Compassionate use of the PASCAL transcatheter mitral valve repair system for patients with severe mitral regurgitation: a multicentre, prospective, observational, first-in-man study. Lancet. 2017;390:773-80.

18. Fam NP, Braun D, von Bardeleben RS, Nabauer M, Ruf T, Connelly KA, et al. Compassionate use of the PASCAL transcatheter valve repair system for severe tricuspid regurgitation: a multicenter, observational, first-in-human experience. JACC Cardiovasc Interv. 2019;12:2488-95.
19. Edwards CLASP TR EFS (CLASP TR EFS). Available at: https://clinicaltrials gov/ct2/show/NCT03745313. Accessed August 19, 2019.

20. Perlman GY, Dvir D. Treatment of tricuspid regurgitation with the FORMA repair system. Front Cardiovasc Med. 2018;5:140.

21. Campelo-Parada F, Perlman G, Philippon F, Ye J, Thompson C, Bédard E, et al. First-in-man experience of a novel transcatheter repair system for treating severe tricuspid regurgitation. J Am Coll Cardiol. 2015;66:2475-83.

22. Nickenig G, Weber M, Schueler R, Hausleiter J, Näbauer M, von Bardeleben RS et al. 6-Month outcomes of tricuspid valve reconstruction for patients with severe tricuspid regurgitation. J Am Coll Cardiol. 2019;73:1905-15.

23. Schueler R, Hammerstingl C, Werner N, Nickenig G. Interventional direct annuloplasty for functional tricuspid regurgitation. JACC Cardiovasc Interv. 2017; 10:415-6.

24. Maisano F, Taramasso M, Nickenig G, Hammerstingl C, Vahanian A, MessikaZeitoun D, et al. Cardioband, a transcatheter surgical-like direct mitral valve annuloplasty system: early results of the feasibility trial. Eur Heart J. 2016;37: 817-25.

25. Kuwata S, Taramasso M, Nietlispach F, Maisano F. Transcatheter tricuspid valve repair toward a surgical standard: first-in-man report of direct annuloplasty with a Cardioband device to treat severe functional tricuspid regurgitation. Eur Heart J. 2017;38:1261.

26. Orban M, Braun D, Orban M, Gross L, Näbauer M, Hagl CM, et al. Established interventions for mitral valve regurgitation. Herz. 2016;41:19-25.

27. Chadwell K, Lim S. Early Feasibility Study of 4D ICE Guidance for Structura Heart TRANSCATHETER interventions. Chicago, IL: TVT 2019; 2019.

28. Millipede I. The Millipede IRIS transcatheter annuloplasty ring. Available at: http://www.millipedemedical.com/iris.html. Accessed August 19, 2019.

29. Rogers JH, Boyd WD, Smith TW, Bolling SF. Early experience with Millipede IRIS transcatheter mitral annuloplasty. Ann Cardiothorac Surg. 2018;7:780-6.

30. Rogers JH. Transcatheter tricuspid valve therapies 5: Millipede. Transcatheter Cardiovasc Ther. 2016

31. Rogers JH. Millipede ring for the tricuspid valve. Transcatheter Cardiovasc Ther 2017.

32. Annular reshaping of the mitral valve for patients with mitral regurgitation using the Millipede IRIS system. Available at: https://clinicaltrials.gov/ct2/show/ NCT02607527. Accessed August 19, 2019.

33. Navia JL, Kapadia S, Elgharably H, Harb SC, Krishnaswamy A, Unai S, et al First-in-human implantations of the NaviGate bioprosthesis in a severely dilated tricuspid annulus and in a failed tricuspid annuloplasty ring. Circ Cardiovasc Interv. 2017;10:e005840.

34. Rotar E, Lim DS, Ailawadi G. Risk stratification for surgery in tricuspid regurgitation. Prog Cardiovasc Dis. 2019;62:500-4. 